\title{
The cosmic-ray electron spectrum measured with H.E.S.S.
}

\author{
Kathrin Egberts ${ }^{* \dagger}$ for the H.E.S.S. Collaboration \\ Max-Planck-Institut für Kernphysik, Heidelberg, P.O. Box 103980, D 69029 Heidelberg, \\ Germany \\ E-mail: kathrin.egberts@uibk.ac.at
}

\begin{abstract}
The measurement of very-high-energy cosmic-ray electrons is intrinsically difficult due to the very steep electron spectrum with low fluxes and an enormous background of hadronic cosmic rays. The large collection areas needed for such a measurement can be provided by ground-based imaging atmospheric Cherenkov telescopes. The High Energy Stereoscopic System (H.E.S.S.) has performed the first ground-based cosmic-ray electron measurement and thereby extended the measured range of the spectrum to several TeV. Here the H.E.S.S. measurement is presented, as well as an extension of the H.E.S.S. spectrum towards lower energies. At these energies, H.E.S.S. can probe recent ATIC measurements, which have been interpreted in terms of dark matter scenarios.
\end{abstract}

European Physical Society Europhysics Conference on High Energy Physics, EPS-HEP 2009, July 16 - 222009

Krakow, Poland

\footnotetext{
*Speaker.

${ }^{\dagger}$ now at Institut für Astro- und Teilchenphysik, Leopold-Franzens-Universität Innsbruck, A-6020 Innsbruck
} 


\section{Introduction}

Electrons are a small and yet highly interesting component of cosmic rays. Due to their low masses they suffer strong energy losses from inverse Compton scattering and synchrotron radiation. These mechanisms restrict the lifetime of a TeV electron according to

$$
t \approx 5 \times 10^{5}\left(\frac{E}{1 \mathrm{TeV}}\right)^{-1}\left(\left(\frac{B}{5 \mu \mathrm{G}}\right)^{2}+1.6\left(\frac{w}{1 \mathrm{eV} \mathrm{cm}^{-3}}\right)\right)^{-1} \text { years }
$$

where $w$ is the photon energy density in the interstellar medium and $B$ is the mean interstellar magnetic field. This limits the number of possible sources of $\mathrm{TeV}$ electrons since only nearby and recent sources contribute to the very-high-energy electron spectrum $[1,2,3]$. This sensitivity to accelerators in our local neighborhood is a unique feature of the cosmic-ray electron spectrum. Furthermore, the cosmic-ray electron spectrum may contain features of dark matter annihilation. Recently, the ATIC collaboration reported the measurement of an excess in the electron spectrum [4]. Combined with the excess in the positron fraction measured by PAMELA [5], the peak feature of the ATIC measurement has been interpreted in terms of a dark matter signal or a contribution of a nearby pulsar (e.g. [6] and references given there). Another consequence of the strong energy losses of cosmic-ray electrons is their steep spectrum, which follows a power-law $d N / d E=k E^{-\Gamma}$ with a spectral index of $\Gamma \approx 3.3$ compared to an index of 2.7 of the spectrum of hadronic cosmic rays. Therefore, with increasing energy, the electron fraction in cosmic rays gets lower and lower, yielding $\approx 0.1 \%$ at $\mathrm{TeV}$ energies. The steep spectrum and low fluxes of cosmic-ray electrons make measurements of cosmic-ray electrons increasingly difficult at higher energies. The spectrum of cosmic-ray electrons in the $\mathrm{GeV}$ range has been measured by balloon or satellite experiments as AMS [7], HEAT [8, 9], BETS [10], PPB-BETS [11], Emulsion Chambers [3], ATIC [4]. However, these measurements are always limited by the physical size of the detector volume and often by limited observation time.

The High Energy Stereoscopic System (H.E.S.S.) [12] has performed the first ground-based measurement of cosmic-ray electrons [13, 14]. The indirect detection of cosmic-ray electrons yields five orders of magnitude larger effective collection areas and is therefore ideally suited for the measurement of the upper end of the cosmic-ray electron spectrum. H.E.S.S. is a system of four imaging atmospheric Cherenkov telescopes situated in the Khomas highland in Namibia. The four telescopes are identical and form a square with $120 \mathrm{~m}$ edges. H.E.S.S. is an experiment designed for the measurement of $>100 \mathrm{GeV} \gamma$ rays. When energetic $\gamma$ rays (as well as electrons and hadronic cosmic rays) hit the Earth's atmosphere they interact with the air molecules and produce a cascade of secondary particles. These secondaries emit Cherenkov radiation, which can be detected from ground. For this purpose, each of the four Cherenkov telescopes is equipped with a $107 \mathrm{~m}^{2}$ mirror, which reflects the Cherenkov light of the air shower into a fine-grained camera of 960 pixels, which records the image.

Because the H.E.S.S. measurement does not discriminate between electrons and positrons, electrons is used generically in the following to refer to both particle and anti-particle. The H.E.S.S. electron analysis presented here is based on the selection of electron-like events in regions far from $\gamma$-ray sources and subtraction of the remaining hadronic cosmic-ray background using air-shower simulations. The simulations are produced with the CORSIKA program [15]. 
Most critical for the ground-based electron measurement is the background determination, which suffers from the lack of information of air showers compared to a well-defined detector volume of a balloon or satellite experiment.

\section{Background Determination}

Since $\gamma$ rays can hardly be distinguished from electrons based on image information of the Cherenkov light, they are avoided in the data selection by making use of their anisotropic nature. In order to avoid a $\gamma$-ray contamination in the data set, data from the direction of any known source of $\gamma$-rays is excluded. Therefore, only observations targeting extragalactic fields with a pointing position of at least $7^{\circ}$ above the Galactic plane are used for the analysis to avoid diffuse $\gamma$-ray emission from the Galactic plane. In the extragalactic data set, any known $\gamma$-ray source (e.g. active galactic nuclei) are amply excluded within a $0.4^{\circ}$ radius. The background of $\gamma$ rays still left in the evaluated data set is the diffuse extragalactic $\gamma$ ray background. It has been measured by EGRET [18] up to $30 \mathrm{GeV}$. As the extragalactic $\gamma$-ray background is strongly affected by pair creation processes on cosmic radiation fields, the prediction of the $\mathrm{TeV}$ flux is subject to large uncertainties. Following [19] its contribution to the cosmic-ray electron flux measured by H.E.S.S. can be estimated to be less than $6 \%$, assuming a blazar spectrum of an unbroken power-law up to $3 \mathrm{TeV}$ with a Gaussian spectral index distribution centered at $\alpha=-2.1$ with $\sigma_{\alpha}=0.35$. The hadronic background can

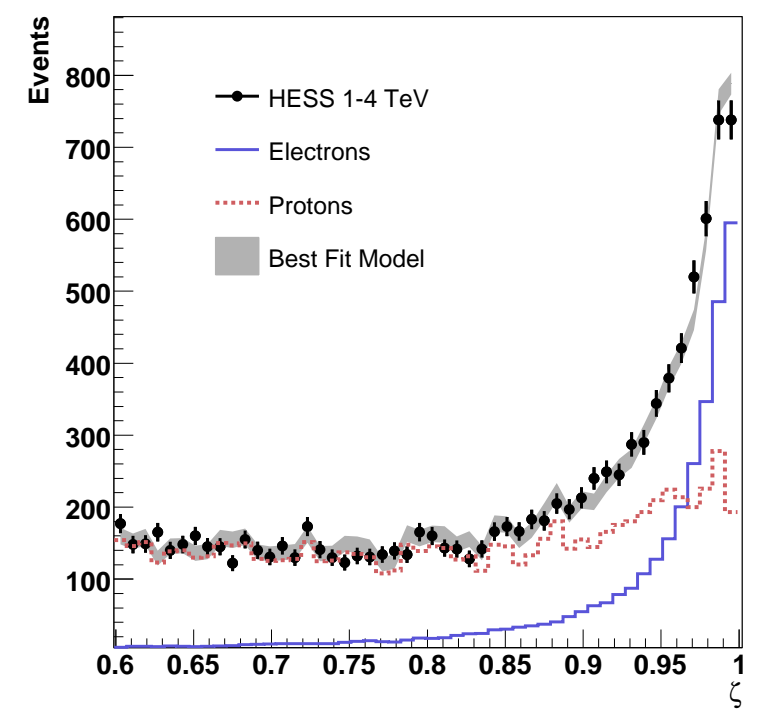

Figure 1: The measured distribution of the parameter $\zeta$, compared with distributions for simulated protons and electrons, for showers with reconstructed energy between 1 and $4 \mathrm{TeV}$. The best fit model combination of electrons and protons is shown as a shaded band. The proton simulations use the SIBYLL hadronic interaction model.

be estimated from the shape of the air shower images in the camera. Hadronic cosmic rays, which interact strongly and electromagnetically in the atmosphere, produce on average broader and irregular showers compared to purely electromagnetically interacting electrons (and $\gamma$-rays). In order to combine any possible image parameters that can contribute to the electron-hadron separation in an optimal way, a machine-learning algorithm is used. The Random Forest algorithm [17] is based on decision trees and is trained with electron simulations and H.E.S.S. data from empty fields. It 
determines the electron likeness $\zeta$ of an event: A $\zeta$ value of zero corresponds to a background event, while a value of one is assigned if the shower is almost certainly an electron. A cut of $\zeta>0.6$ already drastically reduces the hadronic background. The remaining background is estimated by applying a fit in the $\zeta$ distribution. This procedure is visualized in Fig. 1. The H.E.S.S. data are fitted by a model combination of simulated electrons and simulated protons. Thereby, the background estimation makes use of the different shapes of the $\zeta$ distributions of electrons and background events. While electrons (as shown for electron simulations in Fig. 1) exhibit a peak towards $\zeta=1$, the $\zeta$ distribution of the background is basically flat for $\zeta>0.6$. The fit is a two component fit with the free parameters being the number of electrons and the number of protons in the data set. The reason for using simulated protons only for the background component (which also consists of heavier nuclei) is that heavier nuclei have a more background-like appearance and are therefore sufficiently suppressed by the $\zeta>0.6$ cut. This behavior has been tested by simulations. The fitting is applied in independent energy bands and the number of electrons is evaluated. Coupled with the effective collection area for this energy band, the differential flux is calculated.

\section{Results and discussion}

Fig. 2 shows the cosmic-ray electron spectrum determined from H.E.S.S. data together with a compilation of earlier measurements. Shown are two analyses, one optimized for high energies (in blue) and one optimized for low energies (in red). The two analyses differ in the choice of event selection cuts and the data set. For the high-energy analysis, a cut on the image amplitude in each of the four cameras of 200 photo electrons and an impact distance of the shower to the center of the array of less than $200 \mathrm{~m}$ was chosen. The data used were acquired using the complete 4-telescope array during 2004 to 2007 amounting to 239 hours of live-time. The effective collection area using the above described event selection cuts is energy dependent and reaches $\approx 5 \times 10^{4} \mathrm{~m}^{2}$ at $1 \mathrm{TeV}$. The total effective exposure of this data set at $1 \mathrm{TeV}$ is therefore $\approx 8.5 \times 10^{7} \mathrm{~m}^{2} \mathrm{sr}$. For an extension of the spectrum towards lower energies, the analysis has been modified to improve the sensitivity at low energies. In the event selection cuts, the minimum image amplitude has been reduced from 200 to 80 photo electrons to allow for lower energy events. In order to guarantee good shower reconstruction, only events with a reconstructed distance from the projected core position on the ground to the array center of less than $100 \mathrm{~m}$ are included. Additionally, only data taken between 2004 and 2005 are used. The reason is that the H.E.S.S. mirror reflectivity degrades over time and a reduced light yield corresponds to an increased energy threshold. The new data and event selection reduces the event statistics but enables to lower the analysis threshold to $340 \mathrm{GeV}$. The effective collection area at $340 \mathrm{GeV}$ is $\approx 4 \times 10^{4} \mathrm{~m}^{2}$. With a live-time of 77 hours of good quality data, a total effective exposure of $\approx 2.2 \times 10^{7} \mathrm{~m}^{2} \mathrm{srs}$ is achieved at $340 \mathrm{GeV}$. Owing to the steepness of the electron spectrum, the measurement at lower energies is facilitated by the comparatively higher fluxes.

In the region of overlap, the two analyses demonstrate a good agreement. The spectra are parameterized by a power-law in case of the high-energy spectrum and a broken power-law in case of the low-energy spectrum. The high-energy spectrum has a spectral index of $3.9 \pm 0.1_{\text {stat }} \pm 0.3_{\text {syst }}$. The broken power-law starts of with an index of $3.0 \pm 0.1_{\text {stat }} \pm 0.3_{\text {syst }}$ and steepens at $0.9 \pm 0.1 \mathrm{TeV}$ to $4.1 \pm 0.3_{\text {stat }} \pm 0.3_{\text {syst }}$. Systematic errors on the reconstructed spectra arise from uncertainties 


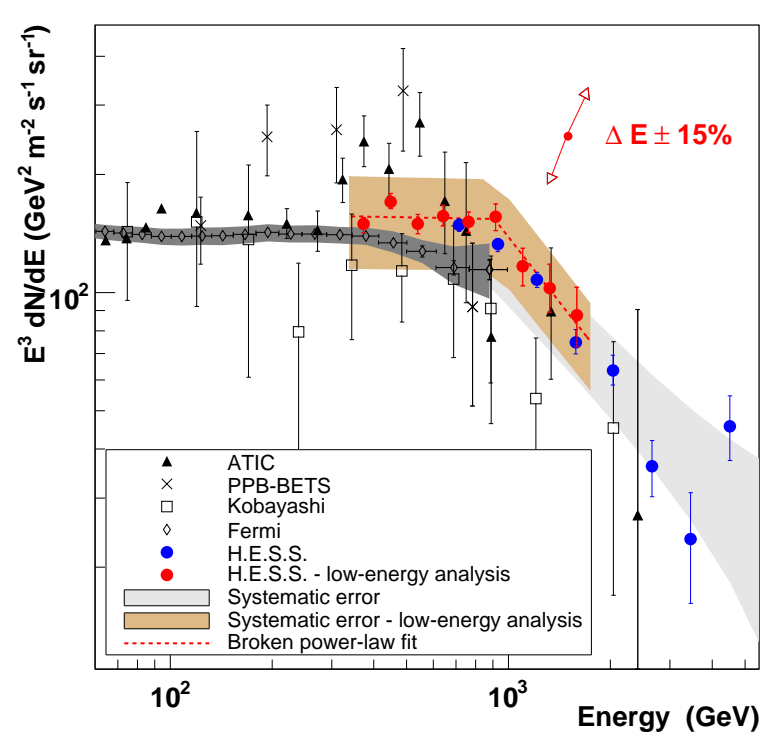

Figure 2: The energy spectrum $\mathrm{E}^{3} \mathrm{dN} / \mathrm{dE}$ of cosmic-ray electrons as measured by H.E.S.S. in comparison with previous measurements. The H.E.S.S. data are shown as solid points. The shaded bands indicate the approximate systematic error arising from uncertainties in the modeling of hadronic interactions and in the atmospheric model. The double arrow indicates the effect of an energy scale shift of $15 \%$, the approximate systematic uncertainty on the H.E.S.S. points. Previous data are reproduced from: AMS [7], HEAT [8], HEAT 94-95 [9], BETS [10], PPB-BETS [11], Kobayashi [3], ATIC [4] and FERMI [20]. The dark grey shaded band indicates the FERMI systematic error.

in the simulation of hadronic interactions and the atmospheric model, as well as in the absolute energy scale. The energy scale uncertainty is $\approx 15 \%$ and is illustrated by a double arrow in Fig. 2. The uncertainty arising from the subtraction of the hadronic background has been estimated by comparison of the spectra obtained with protons simulations of two different hadronic interaction models, namely SIBYLL [21] and QGSJET-II [22]. The effect of atmospheric variations is estimated by a comparison of the spectra obtained by two independent data sets. The effect of latter two uncertainties on the flux normalization are visualized by shaded bands in Fig. 2. The bands are centered around the power-law fits to the data. The systematic error on the spectral indices is $\Delta \Gamma$ (syst.) $\lesssim 0.3$.

The H.E.S.S. measurement reveals a significant steepening of the electron spectrum at higher energies. No indication of an excess and sharp cutoff in the electron spectrum as reported by ATIC is observed. Since H.E.S.S. measures the electron spectrum only above $340 \mathrm{GeV}$, one cannot test the rising section of the ATIC-reported excess. Although different in shape, an overall consistency of the ATIC spectrum with the H.E.S.S. result can be obtained within the uncertainty of the H.E.S.S. energy scale of about $15 \%$. The deviation between the ATIC and the H.E.S.S data is minimal at the $20 \%$ confidence level (assuming Gaussian errors for the systematic uncertainty dominating the H.E.S.S. measurement) when applying an upward shift of $10 \%$ in energy to the H.E.S.S. data. The shift is well within the uncertainty of the H.E.S.S. energy scale. At lower energies, FERMI [20] has recently measured the cosmic-ray electron spectrum between $20 \mathrm{GeV}$ and $1 \mathrm{TeV}$ with unprecedented accuracy. The H.E.S.S. and FERMI measurements demonstrate an excellent agreement 
within the uncertainties of the two instruments. The cosmic-ray electron spectrum as measured by H.E.S.S. and FERMI shows no indication of a Kaluza-Klein dark matter annihilation feature as used to explain the ATIC excess [4]. It is compatible with less pronounced dark matter scenarios as well as conventional electron populations of astrophysical origin within the uncertainties related to the injection spectra and propagation effects.

The support of the Namibian authorities and of the University of Namibia in facilitating the construction and operation of H.E.S.S. is gratefully acknowledged, as is the support by the German Ministry for Education and Research (BMBF), the Max Planck Society, the French Ministry for Research, the CNRS-IN2P3 and the Astroparticle Interdisciplinary Programme of the CNRS, the U.K. Science and Technology Facilities Council (STFC), the IPNP of the Charles University, the Polish Ministry of Science and Higher Education, the South African Department of Science and Technology and National Research Foundation, and by the University of Namibia. We appreciate the excellent work of the technical support staff in Berlin, Durham, Hamburg, Heidelberg, Palaiseau, Paris, Saclay, and in Namibia in the construction and operation of the equipment.

\section{References}

[1] C. S. Shen, Astrophys. J. Lett., 162, L181, (1970).

[2] F. A. Aharonian, A. M. Atoyan, H. J. Völk, Astron. \& Astrophys., 294, L41 (1995).

[3] T. Kobayashi et al., Astrophys. J., 601, 340 (2004).

[4] J. Chang et al., Nature, 456, 362-365 (2008).

[5] O. Adriani et al., Nature, 458, 607-609 (2009).

[6] D. Malyshev, I. Cholis, J. Gelfand arXiv:0903.1310v1 [astro-ph.HE] (2009).

[7] M. Aguilar et al., Physics Reports 366, 331 (2002).

[8] S. W. Barwick et al., Astrophys. J., 498, 779 (1998).

[9] M. A. DuVernois et al., Astrophys. J., 559, 296 (2001).

[10] S. Torii et al., Astrophys. J., 559, 973 (2001).

[11] S. Torii et al., arXiv:0809.0760 [astro-ph] (2008).

[12] J. A. Hinton (H.E.S.S. Collaboration), New Astron. Rev. 48, 331 (2004).

[13] F. A. Aharonian et al., Phys. Rev. Lett. 101, 261104 (2008).

[14] F. A. Aharonian et al., arXiv:0905.0105 [astro-ph] (2009).

[15] D. Heck et al., Forschungszentrum Karlsruhe Report FZKA 6019 (1998).

[16] A. Hillas, Proceedings 19th International Cosmic Ray Conference, La Jolla, F. C. Jones, 3, 445 (1985).

[17] L. Breiman \& A. Cutler, http://www.stat.berkeley.edu/users/breiman/RandomForests/, R. K. Bock et al., Nuclear Instruments and Methods in Physical Research A 516, 511 (2004).

[18] A. W. Strong, O. V. Moskalenko, O. Reimer, Astrophys. J., 613, 956 (2004)

[19] P.S. Coppi \& F. A. Aharonian, Astrophys. J., 487, L9 (1997).

[20] A. A. Abdo et al., Phys. Rev. Lett., 102, 181101 (2009)

[21] R. S. Fletcher, T. K. Gaisser, P. Lipari, \& T. Stanev, Phys. Rev. D 50, 5710 (1994).

[22] S. Ostapchenko et al., Proceedings 29th International Cosmic Ray Conference, Pune, Tata Institute of Fundamental Research, 7, 135 (2005). 